sound measurements between the two patient-specific models for each sensor.

Results Both the Phonocatheter and stethoscope were able to record transluminal and intravascular sounds generated from stenosis respectively. The Phonocather was in good agreement with the stethoscope demonstrating that the peak-to-rms (mean \pm standard deviation) sound amplitude was significantly louder $(\mathrm{p}<0.0001)$ in the TS stenosis region in pre-lumbar puncture model (Stethoscope: $9.03 \pm 1.61$; Phonocatheter: $6.62 \pm 1.55)$ compared to the TS region in post-lumbar puncture model (Stethoscope: $4.20 \pm 0.86$; Phonocatheter: $3.62 \pm 0.88$ ) (figures $1 \mathrm{~B}$ and $1 \mathrm{C}$ ).

Conclusion We have developed a prototype of microphone enabled catheter that can measure sound in patient-specific PT flow models, and potentially measure sounds quantitively in PT patients.

Disclosures M. Amans: 1; C; NIH R01 HL149124-01A1. K. Valluru: None. S. Kondapavulur: None. B. Kilbride: None. H. Haraldsson: None. W. Smith: None. K. Meisel: None. D. Saloner: None.

\section{E-133 DELAYED INTRACEREBRAL HEMORRHAGE AFTER WINGSPAN STENTING FOR INTRACRANIAL INTERNAL CAROTID ARTERY STENOSIS: A CASE REPORT}

1J Kim*, ${ }^{2}$ CHUNG. 'Radiology, Gangnam Severance Hospital, Seoul, Korea, Republic of; ${ }^{2}$ Neurosurgery, Severance Hospital, Seoul, Korea, Republic of

10.1136/neurintsurg-2020-SNIS. 165

Acute ischemic infarction from intracranial atherosclerotic disease accounts for approximately $8 \%-10 \%$ of strokes in the United States each year. At a recent large prospective randomized trial, the efficacy and safety of wingspan stent for intracranial atherosclerotic stenosis was verified when it was used by experienced interventionalist with proper patient selection. Among several procedural complications, delayed hemorrhage is rarely reported. A 45-year-old man presented with a history of dysarthria and recurrent episodes of transient right hemiparesis. The patient was newly found atrial fibrillation and started warfarin medication. Cerebral catheter angiography and single-photon emission computed tomography (SPECT) scan with acetazolamide challenge confirmed moderate (60\%) left carotid artery stenosis and symmetrical findings of both hemispheres. Endovascular Wingspan

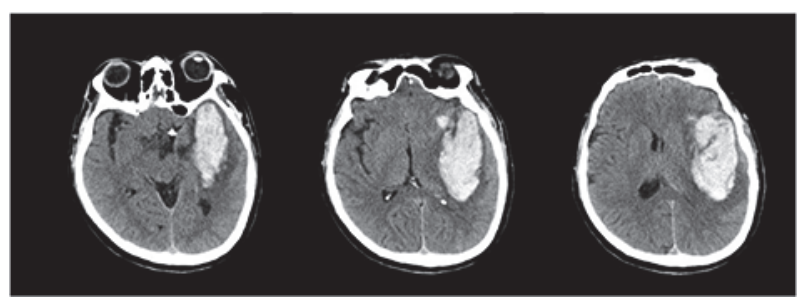

Abstract E-133 Figure 2

stenting of the left communicating segment of internal cerebral artery was performed uneventfully. 22 days after treatment the patient presented with sudden headache and aphasia and CT scan showed intracerebral hemorrhage on left temporal lobe. Due to further rapid clinical deterioration, surgical removal of hematoma was required. The patient's poor neurological outcome did not improve during the 2year follow-up period. This case report illustrates a severe delayed intracerebral hemorrhage following intracranial stenting of the internal carotid artery. The mechanism of delayed hemorrhage is unclear. Currently, 4 mechanisms have been discussed as potential causes of remote ICH after uneventful EVT. These mechanisms are dual antiplatelet therapy, hemorrhagic transformation of clinically silent small periprocedural embolic infarcts, intraprocedural foreign body emboli, and flow modification. This complication should not be neglected because of its poor clinical outcomes.

Disclosures J. Kim: None. J. Chung: None.

\section{E-134 TRANS-ULNAR ACCESS FOR NEUROINTERVENTION: PUSHING THE BOUNDARIES OF ARTERIAL ACCESS}

R Dossani, M Waqas*, M Tso, G Rajah, F Chin, A Yunke, A Siddiqui, J Davies. UB Neurosurgery, Buffalo, NY

\subsection{6/neurintsurg-2020-SNIS. 166}

Background Transradial access has been gaining popularity in neurointerventional surgery. Failure to obtain radial access may lead neurointerventionists to convert to a transfemoral approach, which is associated with a higher complication rate than the radial approach. Ulnar access, however, provides a similar safety profile to radial access and permits neurointerventionists to work in the same hand without having to convert to a femoral approach. In this study, we evaluate the feasibility of using transulnar access to perform diagnostic cerebral angiograms and various neurointerventional procedures.

Methods Consecutive patients who underwent transulnar diagnostic angiogram or neurointerventional procedures were included in the study over a period of 12 months. Data on demographics, procedure indication, devices, technique and complications recorded. A descriptive analysis was carried out.

Results Transulnar access was utilized in 18 patients over the study period. Mean age was $71.6+6.8$ years; $10(55.5 \%)$ patients were male. Fifteen diagnostic angiograms and 3 neurointerventions (1 left middle meningeal artery, 1 right carotid artery stenting, 1 left carotid artery stenting) were performed. All the procedures were performed using a right sided ulnar artery with ultrasound guidance. The indications for ulnar access included a feeble radial artery pulse $(n=17)$, or radial 
artery occlusion due to prior angiogram $(n=1)$. A 5 Fr sheath was used for diagnostic procedures while $6 \mathrm{Fr}$ was used for neurointerventions. A TR band was used for closure. No case required conversion to femoral access. No access related complication was noticed.

Conclusion Transulnar arterial access is safe and feasible for diagnostic and interventional neuroangiography procedures and provides a useful alternative to transradial access, potentially avoiding complications associated with transfemoral access.

Disclosures R. Dossani: None. M. Waqas: None. M. Tso: None. G. Rajah: None. F. Chin: None. A. Yunke: None. A. Siddiqui: None. J. Davies: None.

\section{E-135 ENDOVASCULAR MANAGEMENT OF EPISTAXIS: TIME FOR CONSENSUS? A SYSTEMATIC REVIEW AND META- ANALYSIS}

N Alotaibi ${ }^{*}$, P Harker, F Robertson, J Vranic, M Koch, C Stapleton, T Leslie-Mazwi, A Patel. Neurosurgery, Massachusetts General Hospital, BOSTON, MA

\subsection{6/neurintsurg-2020-SNIS.167}

Background Recently, the American Academy of Otolaryngology-Head and Neck Surgery Foundation (AAO-HNSF) published evidence-based recommendations to improve the quality and management variation for patients who suffer from epistaxis, however, there were no clear guidelines on timing and techniques of endovascular management.

Methods A systematic review and meta-analysis in accordance with the PRISMA (Preferred Reporting Items for Systematic Reviews and Meta-Analyses) guidelines. Our primary outcome was the proportion of patients who had immediate bleeding control after endovascular management. Secondary outcomes included percentage of major adverse events following embolization. Sensitivity analysis was conducted according to center protocol (materials used, unilateral vs. bilateral approach, and timing of treatment).

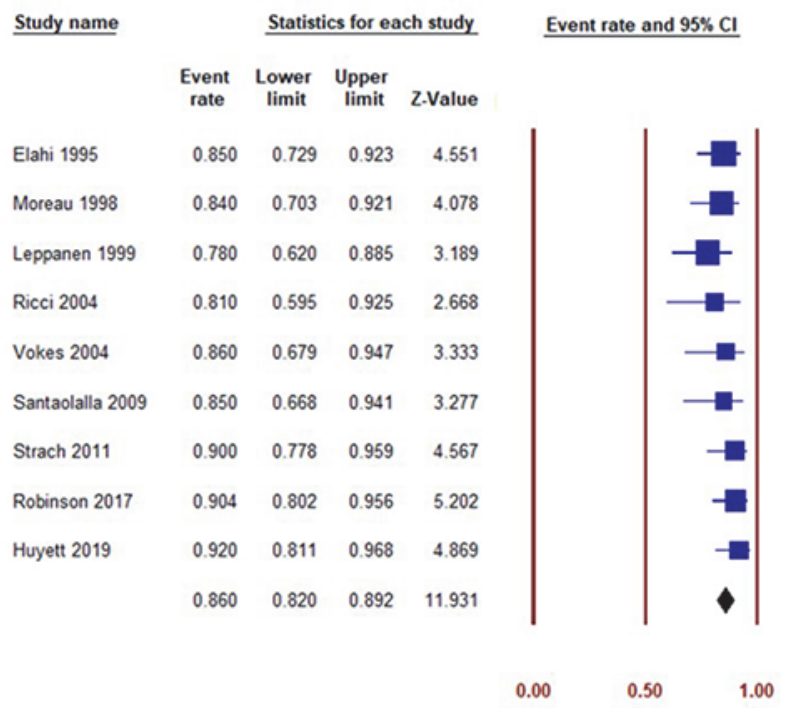

Pooled bleeding control event rates with $95 \% \mathrm{Cl}$

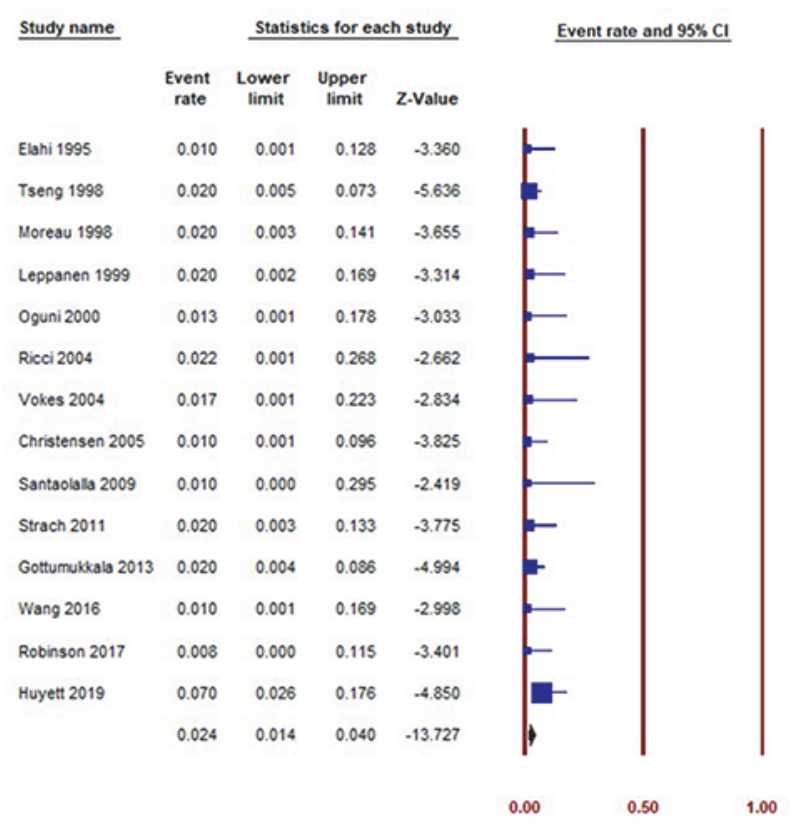

Pooled major complications rates with $95 \% \mathrm{Cl}$

\section{Abstract E-135 Figure 2}

Results Fourteen case series (with 719 patients) met our inclusion criteria for primary and secondary outcome. The pooled event rate for immediate control was $86 \%$ (95\% confidence interval [CI], 82\%-89\%). Major adverse events (stroke, craniofacial skin necrosis, and retro-peritoneal hemorrhage) had an overall event rate of $2.4 \%$ (95\% CI $1.4 \%$ $4 \%)$. Most embolizations were targeting distal portion of the internal maxillary ( $>95 \%$ of cases). Sensitivity analysis revealed that bilateral approaches were associated with less recurrence rates, however, higher rates of major adverse events were notes. Several factors were identified for heterogeneity between studies, including outcomes definitions, follow-up time, angiographic protocol, target vessels and embolization's materials.

Conclusions The best available evidence for endovascular management of epistaxis is based on retrospective observational studies. Deficiency of standardized reporting methods is a significant drawback to understand the exact role of endovascular treatment for epistaxis and whether it is superior to other operative methods.

Disclosures N. Alotaibi: None. P. Harker: None. F. Robertson: None. J. Vranic: None. M. Koch: None. C. Stapleton: None. T. Leslie-Mazwi: None. A. Patel: None.

\section{E-136 SAFETY AND EFFICACY OF AN ACCELERATED DEFLATION ALGORITHM FOR PATENT HEMOSTASIS IN TRANSRADIAL NEUROVASCULAR PROCEDURES}

${ }^{1} \mathrm{~K}$ Mantripragada*, 'S Mansour, 'N Echeverry, 'E Hospedales, 'S Shapiro, ${ }^{2} \mathrm{~B}$ Snelling. ${ }^{1}$ Charles E. Schmidt College of Medicine at Florida Atlantic University, Boca Raton, FL; ${ }^{2}$ Cerebrovascular and Endovascular Neurosurgery, Marcus Neurosciences Institute, Boca Raton, FL

10.1136/neurintsurg-2020-SNIS. 168

Introduction/Purpose Transradial access for neurovascular procedures has gained prominence recently given improved safety 